\title{
Effect of the three-nucleon force on the three-nucleon ground state: Reducible and irreducible contributions
}

\author{
A. Stadler ${ }^{(1)}$ and P. U. Sauer ${ }^{(1,2)}$ \\ ${ }^{(1)}$ Institut für Theoretische Physik, Universität Hannover, D-3000 Hannover 1, Germany \\ ${ }^{(2)}$ Department of Physics, FM-15, University of Washington, Seattle, Washington 98195
}

(Received 5 March 1992)

\begin{abstract}
The Tucson-Melbourne two-pion exchange three-nucleon force is split into a reducible part mediated by $\Delta$ excitation and an irreducible part not mediated by $\Delta$ excitation. The triton binding energy is calculated. The irreducible part of the Tucson-Melbourne force is taken into account in the calculation. Its reducible part is discarded, but regenerated by the explicit treatment of the $\Delta$ isobar in a coupledchannel approach. The remaining irreducible part of the Tucson-Melbourne force makes a nonnegligible contribution to the triton binding energy. The strong dependence of the triton binding energy on the cutoff parameter for the $\pi N N$ vertex in the Tucson-Melbourne force is considerably reduced.
\end{abstract}

PACS number(s): 21.30. $+\mathrm{y}, 21.45 .+\mathrm{v}, 25.10 .+\mathrm{s}$

\section{INTRODUCTION}

There are two different strategies for describing the three-nucleon force in nuclear systems. Both strategies have been tried out in accurate calculations for the three-nucleon bound state. The first strategy [1-3] employs a purely nucleonic Hilbert space and adds an irreducible three-nucleon potential to a traditional Hamiltonian consisting of two-nucleon potentials. The second strategy $[4,5]$ assumes that the excitation of a $\Delta$ isobar in the nuclear medium is the dominant mechanism for the effective three-nucleon force. It extends the Hilbert space to include single $\Delta$-isobar configurations explicitly and thereby builds up the three-nucleon force from simpler two-particle interactions. When applied to the threenucleon bound state, both strategies see strikingly different effects of the three-nucleon force on the binding energy. In the first strategy [1-3], the effect is large and sensitively depends on the regularization of the threenucleon force at small relative distances. The regularization parameter can be chosen as fit parameter to allow the theoretical binding-energy prediction to come in agreement with the experimental value. In the second strategy $[4,5]$, the effect on the binding energy is comparatively small. Explicit $\Delta$-isobar excitation yields competing processes [5]. Besides an attractive three-nucleon force, it yields a weakening of the two-nucleon attraction, i.e., a repulsion of comparable magnitude.

Both approaches have advantages and disadvantages: (1) The first strategy may easily accommodate contributions from diverse processes in the parametrization of the three-nucleon force. But the contributing processes are frozen and cannot adjust to the nuclear medium. Furthermore, the added phenomenology is hardly made consistent in the employed two- and three-nucleon potentials. (2) The second strategy can connect the $\Delta$-isobar excitation to the mechanism of pion production [6] in the two-nucleon system above the pion threshold and, in this aspect, could achieve consistency between the two- and three-nucleon contributions. However, other physics processes contributing to the three-nucleon force besides the $\Delta$-isobar excitation are left out altogether.

The present paper tries to unify both approaches to the three-nucleon force. It uses the second strategy and therefore treats the $\Delta$-isobar degree of freedom explicitly. But it also adds a three-nucleon potential to the Hamiltonian in order to account for those processes not mediated by $\Delta$-isobar excitation. It presents the first calculation of the three-nucleon binding energy within that unifying calculational scheme.

Section II discusses the two-pion exchange TucsonMelbourne three-nucleon force usually employed in the first strategy. It describes how we take out the process of $\Delta$-isobar excitation from that force. Section III recalls the calculational apparatus for solving the Faddeev equations in momentum space in the presence of a threenucleon potential. Section IV gives results and conclusions.

\section{TUCSON-MELBOURNE THREE-NUCLEON FORCE AND $\triangle$-ISOBAR EXCITATION}

The Tucson-Melbourne three-nucleon force [7] is based on pion exchange. Its main ingredient is the pionnucleon scattering amplitude with the pion off mass shell, whereas the nucleon is assumed to remain on shell. Since the experimental information refers to on-shell pionnucleon scattering only, Ref. [7] has to perform an offshell extrapolation of the scattering amplitude; it uses constraints originating from current algebra and the theory of partially conserved axial-vector current (PCAC) in order to minimize the model dependence in that extrapolation. Figure 1 shows processes contributing to the Tucson-Melbourne force. The contribution arising from the nucleon-pole term in the pion-nucleon scattering amplitude according to Fig. 1(b) is also generated by the iterated one-pion exchange two-nucleon force between three nucleons; it is therefore subtracted and not retained 
in the Tucson-Melbourne three-nucleon force. The inclusion of rho exchange into the Tucson-Melbourne three-nucleon force is presently considered [8].

The resulting three-nucleon potential $W$ has three equivalent pieces $W_{i}, i$ denoting that nucleon which simultaneously interacts with the two others, i.e.,

$$
W=\sum_{i} W_{i}
$$

In momentum space the potential reads $[9,10]$

$$
\begin{aligned}
\left\langle\mathbf{k}_{1}^{\prime} \mathbf{k}_{2}^{\prime} \mathbf{k}_{3}^{\prime}\left|W_{1}\right| \mathbf{k}_{1} \mathbf{k}_{2} \mathbf{k}_{3}\right\rangle= & \frac{1}{(2 \pi)^{6}} \delta\left(\mathbf{k}_{1}^{\prime}+\mathbf{k}_{2}^{\prime}+\mathbf{k}_{3}^{\prime}-\mathbf{k}_{1}-\mathbf{k}_{2}-\mathbf{k}_{3}\right) \frac{g^{2}}{4 m_{N}^{2}} \frac{\sigma_{2} \cdot \mathbf{Q}}{\mathbf{Q}^{2}+\mu^{2}} \frac{\sigma_{3} \cdot \mathbf{Q}^{\prime}}{\mathbf{Q}^{\prime 2}+\mu^{2}} H\left(\mathbf{Q}^{2}\right) H\left(\mathbf{Q}^{\prime 2}\right) \\
& \times\left\{\tau_{2} \cdot \tau_{3}\left[a+b \mathbf{Q} \cdot \mathbf{Q}^{\prime}+c\left(\mathbf{Q}^{2}+\mathbf{Q}^{\prime 2}\right)\right]+d\left(\tau_{1} \cdot \tau_{3} \times \tau_{2}\right)\left(\sigma_{1} \cdot \mathbf{Q} \times \mathbf{Q}^{\prime}\right)\right\}
\end{aligned}
$$

where $\mathbf{k}_{i}, \sigma_{i}$, and $\tau_{i}$ are momentum, spin, and isospin of nucleon $i, m_{N}=938.92 \mathrm{MeV}$ is the nucleon mass, $\mu=139.6 \mathrm{MeV}$ the mass of the charged pions, and $g^{2} / 4 \pi=197.7 / 4 \pi=15.73$ the pseudoscalar $\pi N N$ coupling constant. The vertex form factors depend on the pion three-momenta $\mathbf{Q}=\mathbf{k}_{2}-\mathbf{k}_{2}^{\prime}$ and $\mathbf{Q}^{\prime}=\mathbf{k}_{3}^{\prime}-\mathbf{k}_{3}$ and are chosen to be of dipole form, i.e.,

$$
H\left(\mathbf{Q}^{2}\right)=\left(\frac{\Lambda^{2}-\mu^{2}}{\Lambda^{2}+Q^{2}}\right)^{2}
$$

The pion-nucleon scattering amplitude on which the Tucson-Melbourne three-nucleon force is based is not partial-wave projected. Nevertheless, the strength parameters $a$ and $c$ in Eq. (2) are controlled by $S$-wave pion-nucleon scattering, the parameters $b$ and $d$ by $P$ wave scattering. A three-nucleon potential of the same structure is derived in Ref. [11] using an effective chiralinvariant Lagrangian.

How much of the strength of the Tucson-Melbourne three-nucleon force is due to the resonance in $P_{33}$ pionnucleon scattering? This is the same question with respect to the $\Delta$ isobar as Ref. [7] posed and answered for the nucleon-pole contribution of Fig. 1(b) in the original derivation. In the same spirit we want to subtract the $\Delta$ pole contribution of Fig. 1(a) from the Tucson-Melbourne three-nucleon force. We need such a subtraction, since we prefer to treat the excitation of the nucleon to a $\Delta$ iso-

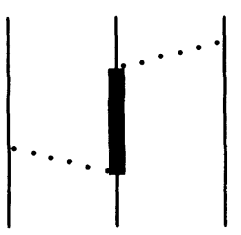

(a)

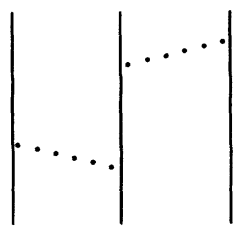

(b)

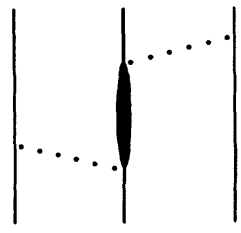

(c)
FIG. 1. Processes which are contained in the TucsonMelbourne two-pion exchange three-nucleon force. The solid lines represent nucleons, the dotted lines pions. The thick line in the middle of (a) stands for the resonant contribution to the $P_{33}$ pion-nucleon scattering amplitude, (b) describes the $P_{11}$ nucleon-pole contribution, and the dark blob in the middle of (c) stands for the remaining pion-nucleon scattering without $P_{33}$ resonance and $P_{11}$ nucleon pole. All time orders of pion exchange are considered in their contributions to the threenucleon force. The pion-nucleon scattering amplitude on which the Tucson-Melbourne three-nucleon force is based is not split up into the processes (a)-(c). That conceptual split is, however, convenient for the purposes of this paper. bar explicitly according to the coupled-channel approach of Refs. [4,5]. In that coupled-channel approach, the purely nucleonic Hilbert space is extended by a sector in which one nucleon is replaced by a $\Delta$ isobar. The $\Delta$ isobar is excited through an instantaneous two-baryon transition potential from two-nucleon to nucleon- $\Delta$ states. The force model previously employed in Refs. [4,5] consists of the processes (a)-(d) in Fig. 2, (b) standing for

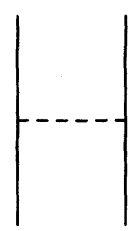

(a)

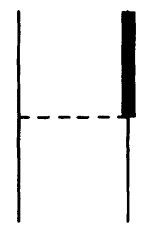

(b)

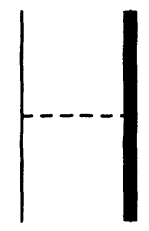

(c)

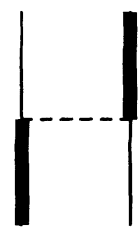

(d)

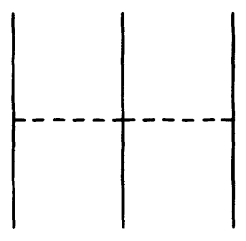

(e)

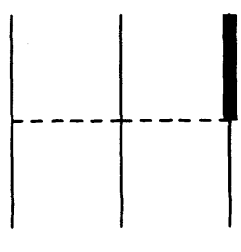

(f)

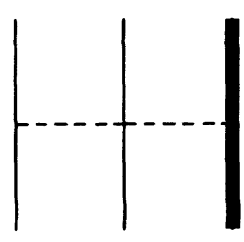

(g)

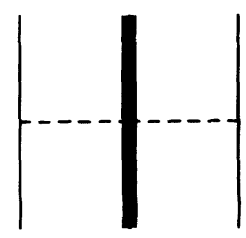

(h)

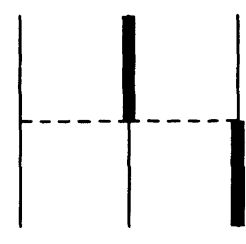

(i)

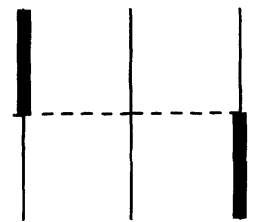

(j)
FIG. 2. Force model in the extended Hilbert space with explicit $\Delta$-isobar degrees of freedom. Solid lines represent nucleons, thick lines $\Delta$ isobars. The horizontal dashed lines stand for instantaneous potentials. Diagram (e) indicates an irreducible three-nucleon force; diagrams $(f)-(j)$ indicate irreducible three-baryon forces connected with additional $\Delta$-isobar excitation. 
that transition potential. The force model generates effective contributions to the two- and three-nucleon interactions. Characteristic pieces are shown in Fig. 3. The effective three-nucleon force of Fig. 3(a) corresponds to the contribution in the Tucson-Melbourne threenucleon force arising from the $P_{33}$ pion-nucleon resonance according to Fig. 1(a). Thus, whenever the Tucson-Melbourne three-nucleon force is added to a coupled-channel force model with $\Delta$-isobar excitation, that $\Delta$-pole part has to be removed from the TucsonMelbourne force-in the same way as its nucleon-pole part had to be removed when two- and three-nucleon forces were combined in a nuclear Hamiltonian. We therefore need a subtraction scheme for the $\Delta$-pole part.

One may either subtract the $P_{33}$ resonance contribution in the off-shell pion-nucleon amplitude from which the Tucson-Melbourne three-nucleon force is derived or one may leave out a part of the Tucson-Melbourne three-nucleon force equivalent in magnitude to what a force model with $\Delta$-isobar excitation yields in the proposed coupled-channel approach anyhow. Provided the coupling parameters between $\Delta$ isobar and pion are taken to be the same and the static approximation on the $\Delta$ propagator is made in an identical fashion, either subtraction scheme has to yield the same $\Delta$-pole contribution to the three-nucleon force.

(1) The coupled-channel approach of Refs. [4,5] is based on the processes (a)-(d) in the force model with single $\Delta$-isobar excitation defined in Fig. 2 . Its transition potential (b) contains pion and rho exchange. Thus, in contrast to Ref. [7], the effective three-nucleon force of Fig. 3(a) contains pion and rho exchange. The pionexchange part of the transition potential yields - in instantaneous and static approximation-precisely a pionexchange three-nucleon force with the form of Eq. (2). For example, the particular transition potential in the force model Al of Refs. [5,12] gives the particular strength parameters $a_{\Delta 1}=c_{\Delta 1}=0, b_{\Delta 1}=-1.83 \mu^{-3}$, and $d_{\Delta 1}=b_{\Delta 1} / 4=-0.46 \mu^{-3}$. In a Tucson-Melbourne force, in which a part equivalent to the one generated by the iteration of that particular transition potential as in Fig. $3(a)$ is left out, the strength parameters are to be reduced by the indicated amounts. The resulting values are given in line 2 of Table I as TM- $\Delta 1$.

(2) The pion-nucleon scattering amplitude of Ref. [7] is not partial-wave projected. Thus the amplitude contribu-

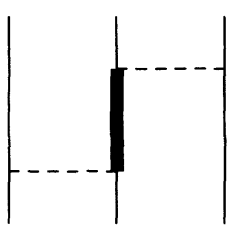

(a)

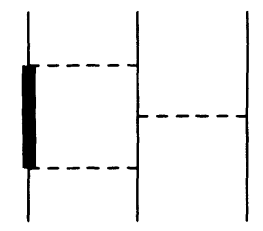

(b)

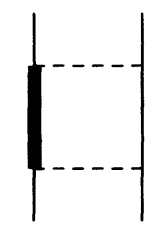

(c)
FIG. 3. Examples for effective two- and three-nucleon processes generated by the two-baryon part of the force model defined in Fig. 2. The processes (a) and (b) contribute to the effective three-nucleon force; the process (c) yields a modification of the two-nucleon interaction in the three-nucleon system. tion arising from the $P_{33}$ partial wave is neither split into resonance and background nor even made explicit at all. Nevertheless, according to archaeological efforts [13], Ref. [7] assumed coupling parameters which would yield the $P_{33}$ resonance contributions $a_{\Delta 2}=c_{\Delta 2}=0$, $b_{\Delta 2}=-1.48 \mu^{-3}$, and $d_{\Delta 2}=-0.35 \mu^{-3}$ to the strength parameters in the Tucson-Melbourne force. In a Tucson-Melbourne force without that $P_{33}$ resonance contribution, the strength parameters are to be reduced by the indicated amounts. The resulting values are given in line 3 of Table I as TM- $\Delta 2$.

The resulting strength parameters of the TucsonMelbourne two-pion exchange three-nucleon force, from which the $\Delta$-pole contribution is subtracted, are different, since the coupling strength between the $\Delta$ isobar and the pion is assumed to be different in the two subtraction schemes.

This paper calculates the triton binding energy for the force model of Fig. 2. By process (e) the calculation includes a three-nucleon force irreducible in a Hilbert space with $\Delta$ isobars. The Tucson-Melbourne pion-exchange three-nucleon force, from which the $\Delta$-pole contribution is subtracted according to either of the discussed schemes, is employed as such an irreducible threenucleon force. Irreducible three-baryon forces connected with $\Delta$-isobar excitation according to processes $(f)-(j)$ in Fig. 2 are still left out. The calculation unifies previously distinct approaches to the treatment of a three-nucleon force in the three-nucleon system: (i) It allows the $\Delta$ isobar its proper propagation in the nuclear medium as in Refs. $[4,5]$. (ii) It simultaneously preserves the full physical richness of a three-nucleon force which is based on other contributions besides single $\Delta$-isobar excitation as the Tucson-Melbourne force of Ref. [7] is.

Such a unification of the two distinct approaches to the three-nucleon force is pressing, since so far they have yielded remarkably different results in calculations: The addition of the Tucson-Melbourne pion-exchange threenucleon force to a Hamiltonian with two-nucleon interactions provided [1-3] ample additional binding for the triton. In contrast, the explicit treatment of the $\Delta$ isobar also modified the effective two-nucleon interaction according to Fig. 3(c), thereby balancing off part of the attraction due to the $\Delta$-mediated three-nucleon force; a comparatively small net increase in triton binding [5] resulted. The unification of the two distinct approaches suggested in this paper preserves the respective advantages of both approaches, discussed in the Introduction.

TABLE I. Strength parameters $a, b, c$, and $d$ in TucsonMelbourne-type two-pion exchange three-nucleon forces of Eq. (2). The first line gives the strength values of the original parametrization [7]; the second and third lines give the remaining strength values, once the $\Delta$ contribution is subtracted according to the distinct coupling parameters in the subtraction schemes (1) and (2) of Sec. II.

\begin{tabular}{lcccc}
\hline \hline & $\mu \mathrm{a}$ & $\mu^{3} \mathrm{~b}$ & $\mu^{3} \mathrm{c}$ & $\mu^{3} \mathrm{~d}$ \\
\hline TM & 1.130 & -2.580 & 1.000 & -0.753 \\
TM- $\Delta 1$ & 1.130 & -0.750 & 1.000 & -0.296 \\
TM- $\Delta 2$ & 1.130 & -1.100 & 1.000 & -0.403 \\
\hline \hline
\end{tabular}




\section{CALCULATIONAL PROCEDURE}

The calculational technique of Ref. [14] is adopted and extended to include $\Delta$-isobar excitation. The homogeneous integral equation to be solved for the Faddeev amplitude $|\psi\rangle$ of the three-nucleon bound state is

$$
|\psi\rangle=G_{0}(E)\left\{T(E) P+\left[1+T(E) G_{0}(E)\right] W(1+P)\right\}|\psi\rangle .
$$

In Eq. (4), $G_{0}(E)$ is the free three-baryon Green's function, $T(E)$ the two-baryon transition matrix, $W$ the irreducible three-nucleon potential, $P$ the sum of the cyclic and anticyclic permutation operators, and $E$ the boundstate energy. The quantities $|\psi\rangle, T(E)$, and $W$ should carry an index indicating the spectating baryon which does not participate in the two-baryon interaction of $T(E)$ and simultaneously indicating the nucleon which interacts with both others in the three-nucleon potential $W$ according to Eq. (1). That index is suppressed: The three equations for the three Faddeev amplitudes have all the same form.

The integral equation (4) is solved in momentum space. The momentum space basis states $|p q v\rangle$ are defined as in Refs. [5,14]. Purely nucleonic channels and channels with one $\Delta$ isobar instead of a nucleon are considered. The momentum $p$ refers to the relative motion of the particles in the pair and the momentum $q$ to the motion of the spectator particle relative to the center of mass of the pair. The label $v$ stands for the discrete quantum numbers $[(L S) I(l s) j] \not \mathcal{\partial}_{z}(T t) \mathcal{T} \mathcal{T}_{z} B b$, differentiating the various three-particle channels. The discrete quantum numbers $L S I T B$ denote in turn the orbital angular momentum, spin, total angular momentum, isospin, and baryon content of the pair, with $B=1$ indicating a two- nucleon state and $B=0$ a nucleon- $\Delta$ state. The discrete quantum numbers $l s j t b$ denote in turn the orbital angular momentum, spin, angular momentum, isospin, and baryon character of the spectating baryon, with $b=\frac{1}{2}$ indicating a nucleon and $b=-\frac{1}{2}$ a $\Delta$ isobar. The $(I j)$ coupling scheme is used. The quantum numbers $\not \mathscr{C}_{z} \mathcal{T} \mathcal{T}_{z}$ denote the total angular momentum with projection and the total isospin with projection of the three-nucleon bound state. The basis states are not fully antisymmetrized; they are antisymmetrized with respect to the particles in the pair. The three-particle basis states taken into account are chosen according to the discrete quantum numbers of the pair which correspond to the partial waves of the two-baryon interaction retained as nonvanishing in the actual calculation.

The three-particle momentum space matrix elements of the two-baryon transition matrix $T(E)$, of the threenucleon potential $W$, and of the permutation operator $P$ are defined as in Ref. [14]. We only repeat the matrix elements of the two-baryon transition matrix $T(E)$, i.e.,

$$
\left\langle p q v|T(E)| p^{\prime} q^{\prime} v^{\prime}\right\rangle=\frac{\delta\left(q-q^{\prime}\right)}{q^{2}} t_{v v^{\prime}}\left(p, p^{\prime} ; E, q\right),
$$

where the simplifying Kronecker deltas for the quantum numbers $\not \partial_{z} \mathcal{T} \mathcal{T}_{z} I T l s j t b$ conserved in the two-body interaction are not made explicit. The dependence of the transition matrix on the available energy $E$ and on the spectator momentum $q$ cannot be cast into a single channel-independent form $E-q^{2} / 2 M$ as in Ref. [14]. The matrix elements of the permutation operator $P$ are given in the Appendix. Compared with Ref. [14], they are generalized for the mass difference between a nucleon and a $\Delta$ isobar.

After partial-wave decomposition the integral equation (4) takes the form

$$
\begin{aligned}
& \langle p q v \mid \psi\rangle=\frac{1}{E-p^{2} / 2 \mu_{v}-q^{2} / 2 M_{v}} \\
& \times\left\{\sum_{v^{\prime} v^{\prime \prime}} \int_{0}^{\infty} d q^{\prime} q^{\prime 2} \int_{-1}^{+1} d x t_{v v^{\prime \prime}}\left(p, p_{1}^{v^{\prime \prime} v^{\prime}}\left(q, q^{\prime}, x\right) ; E, q\right)\right. \\
& \times \frac{G_{v^{\prime \prime} v^{\prime}}\left(q, q^{\prime}, x\right)}{\left[p_{1}^{v^{\prime \prime} v^{\prime}}\left(q, q^{\prime}, x\right)\right]^{L^{\prime \prime}}\left[p_{2}^{v^{\prime \prime} v^{\prime}}\left(q, q^{\prime}, x\right)\right]^{L^{\prime}}}\left\langle p_{2}^{v^{\prime \prime} v^{\prime}}\left(q, q^{\prime}, x\right) q^{\prime} v^{\prime} \mid \psi\right\rangle+\sum_{v^{\prime}} \int_{0}^{\infty} d p^{\prime} p^{\prime 2} \int_{0}^{\infty} d q^{\prime} q^{\prime 2} W_{v v^{\prime}}\left(p q, p^{\prime} q^{\prime}\right)\left\langle p^{\prime} q^{\prime} v^{\prime} \mid \psi\right\rangle \\
& +\sum_{v^{\prime} v^{\prime \prime}} \int_{0}^{\infty} d q^{\prime \prime} q^{\prime \prime 2} \int_{0}^{\infty} d q^{\prime} q^{\prime 2} \int_{-1}^{+1} d x W_{v v^{\prime \prime}}\left(p q, p_{1}^{\nu^{\prime \prime} v^{\prime}}\left(q^{\prime \prime}, q^{\prime}, x\right) q^{\prime \prime}\right) \\
& \times \frac{G_{v^{\prime \prime} v^{\prime}}\left(q^{\prime \prime}, q^{\prime}, x\right)}{\left[p_{1}^{v^{\prime \prime} v^{\prime}}\left(q^{\prime \prime}, q^{\prime}, x\right)\right]^{L^{\prime \prime}}\left[p_{2}^{v^{\prime \prime} v^{\prime}}\left(q^{\prime \prime}, q^{\prime}, x\right)\right]^{L^{\prime}}}\left\langle p_{2}^{v^{\prime \prime} v^{\prime}}\left(q, q^{\prime}, x\right) q^{\prime} v^{\prime} \mid \psi\right\rangle \\
& +\sum_{v^{\prime} v^{\prime \prime}} \int_{0}^{\infty} d p^{\prime \prime} p^{\prime \prime 2} \int_{0}^{\infty} d p^{\prime} p^{\prime 2} \int_{0}^{\infty} d q^{\prime} q^{\prime 2} t_{v v^{\prime \prime}}\left(p, p^{\prime \prime} ; E, q\right) \frac{1}{E-p^{\prime \prime 2} / 2 \mu_{v^{\prime \prime}}-q^{2} / 2 M_{v}} W_{v^{\prime \prime} v^{\prime}}\left(p^{\prime \prime} q, p^{\prime} q^{\prime}\right)\left\langle p^{\prime} q^{\prime} v^{\prime} \mid \psi\right\rangle \\
& +\sum_{v^{\prime} v^{\prime \prime} v^{\prime \prime \prime}} \int_{0}^{\infty} d p^{\prime \prime} p^{\prime \prime 2} \int_{0}^{\infty} d q^{\prime \prime \prime} q^{\prime \prime \prime 2} \int_{0}^{\infty} d q^{\prime} q^{\prime 2} \int_{-1}^{+1} d x t_{v v^{\prime \prime}}\left(p, p^{\prime \prime} ; E, q\right) \\
& \times \frac{1}{E-p^{\prime \prime 2} / 2 \mu_{v^{\prime \prime}}-q^{2} / 2 M_{v}} W_{v^{\prime \prime} v^{\prime \prime \prime}}\left(p^{\prime \prime} q, p_{1}^{\nu^{\prime \prime \prime} v^{\prime}}\left(q^{\prime \prime \prime}, q^{\prime}, x\right) q^{\prime \prime \prime}\right) \\
& \left.\times \frac{G_{v^{\prime \prime \prime} v^{\prime}}\left(q^{\prime \prime \prime}, q^{\prime}, x\right)}{\left[p_{1}^{v^{\prime \prime \prime} v^{\prime}}\left(q^{\prime \prime \prime}, q^{\prime}, x\right)\right]^{L^{\prime \prime \prime}}\left[p_{2}^{v^{\prime \prime \prime} v^{\prime}}\left(q^{\prime \prime \prime}, q^{\prime}, x\right)\right]^{L^{\prime}}}\left\langle p_{2}^{v^{\prime \prime \prime} v^{\prime}}\left(q^{\prime \prime \prime}, q^{\prime}, x\right) q^{\prime} v^{\prime} \mid \psi\right\rangle\right\} \text {. }
\end{aligned}
$$


TABLE II. Triton binding energy in MeV obtained from the RSC and Paris two-nucleon potentials with a three-nucleon force added. The results refer to calculations with 5 and 18 purely nucleonic channels. For the results of the first column, no three-nucleon force is included in the calculation. In the additional columns results are compared for the three-nucleon force of Eq. (2), i.e., for the full Tucson-Melbourne (TM) three-nucleon force [7] and for the Tucson-Melbourne force without a $\Delta$-pole contribution (TM- $\Delta 1$ ), that contribution subtracted according to the procedure (1) of Sec. II. The three-nucleon forces are employed with various cutoff parameters $\Lambda$ in the $\pi N N$ vertex of Eq. (3).

\begin{tabular}{lrcccccrr}
\hline & & \multicolumn{2}{c}{$\Lambda=4.1 \mu$} & \multicolumn{2}{c}{$\Lambda=5.8 \mu$} & \multicolumn{2}{c}{$\Lambda=7.1 \mu$} \\
& & No TM & TM & TM- 1 & TM & TM- 1 & TM & TM- $\Delta 1$ \\
\hline RSC & 5 & -7.02 & -6.95 & -6.82 & -7.56 & -7.09 & -8.76 & -7.87 \\
RSC & 18 & -7.23 & -7.45 & -7.22 & -8.92 & -7.73 & -11.36 & -8.50 \\
Paris & 5 & -7.30 & -7.24 & -7.07 & -8.14 & -7.54 & -11.16 & -11.83 \\
Paris & 18 & -7.38 & -7.55 & -7.34 & -9.08 & -7.92 & -12.37 & -9.26 \\
\hline \hline
\end{tabular}

Many quantities in Eq. (6) contain the reduced mass $\mu_{v}$ of the interacting pair and the reduced mass $M_{v}$ of the spectator and the pair c.m. The reduced masses depend on the baryonic content $B b$ of channel $v$, i.e., they are different for purely nucleonic channels and for channels with a $\Delta$ isobar. In the latter case additional differences arise, if the $\Delta$ isobar belongs to the pair or if it is the spectator particle. The momenta $p_{1}^{v v^{\prime}}$ and $p_{2}^{v v^{\prime}}$ are defined in the Appendix; they depend on the relative momenta and reduced masses of two channels $v$ and $v^{\prime}$ and therefore have to carry both channel labels.

The integral equation (6) is the numerical problem of this paper. It is solved by the techniques of Ref. [14].

\section{RESULTS}

Before discussing the unifying calculations for the triton binding energy, results of a reference calculation with purely nucleonic interactions are presented. In that reference calculation the Reid soft-core (RSC) [15] and Paris [16] potentials are used as two-nucleon interactions. The Tucson-Melbourne two-pion exchange three-nucleon force is added with different parameter specifications. The three-nucleon force is employed as full force in the original parametrization, our results of which are already given in Ref. [14]. It is also employed without the $\Delta$-pole contribution, that contribution subtracted according to the two schemes of Sec. II based on distinct coupling pa- rameters. The corresponding results are listed in Tables II and III as TM- $\Delta 1$ and TM- $\Delta 2$, respectively. The results refer to calculations based on 5 and 18 threenucleon channels in the basis of Sec. III. In the 5channel calculations, interactions in all two-nucleon partial waves of positive parity with total two-nucleon angular momentum $I \leq 1$ are taken into account and, in the 18-channel calculations, interactions in all two-nucleon partial waves of either parity with total two-nucleon angular momentum $I \leq 2$. Only the 18 -channel calculations can be considered as rather well converged; the 5-channel calculations were useful for the authors of this paper to spot trends of results. They are also published, since they may be useful for others interested in repeating and extending the calculations of this paper.

The reference calculations of Tables II and III confirm the result of Ref. [17] that a substantial amount of the additional triton binding arising from the inclusion of the Tucson-Melbourne pion-exchange three-nucleon force is due to the $\Delta$-pole contribution, but also that the TucsonMelbourne force without its $\Delta$-pole part still makes a non-negligible contribution. That contribution depends on the cutoff parameter $\Lambda$ for the $\pi N N$ vertex in the three-nucleon force of Eq. (2). The results for the Reid soft-core and Paris potentials show a quantitatively similar behavior with respect to variations of the cutoff parameter $\Lambda$. The only exception is a marked difference between the two potentials in the 5-channel calculations for

TABLE III. Triton binding energy in MeV obtained from the RSC and Paris two-nucleon potentials with a three-nucleon force added. The results refer to calculations with 5 and 18 purely nucleonic channels. For the results of the first column, no three-nucleon force is included in the calculation. In the additional columns results are compared for the three-nucleon force of Eq. (2) and Table I, i.e., for the full Tucson-Melbourne (TM) three-nucleon force [7] and for the Tucson-Melbourne force without a $\Delta$-pole contribution (TM- $\Delta 1$ and TM- $\Delta 2$ ), that contribution subtracted according to the procedures (1) and (2) of Sec. II. The three-nucleon forces are employed with the cutoff parameter $\Lambda=5.8 \mu$ in the $\pi N N$ vertex of Eq. (3).

\begin{tabular}{lrcccc}
\hline \hline & & & $\Lambda=5.8 \mu$ & \\
& & No TM & TM & TM- 1 & TM- $\Delta 2$ \\
\hline RSC & 5 & -7.02 & -7.56 & -7.09 & -7.15 \\
RSC & 18 & -7.23 & -8.92 & -7.73 & -7.92 \\
Paris & 5 & -7.30 & -8.14 & -7.54 & -7.62 \\
Paris & 18 & -7.38 & -9.08 & -7.92 & -8.10 \\
\hline \hline
\end{tabular}


TABLE IV. Two-baryon partial waves ${ }^{(2 S+1)} L_{I}$ taken into account in the solution of Eq. (4). Nucleon- $\Delta$ states couple to two-nucleon states only for a total pair isospin of $T=1$. The $N \Delta$ partial waves in parenthesis have $L \geq 3$. The coupling to those partial waves is included in the calculation of the twobaryon transition matrices, but they are excluded from the basis of three-particle states [5].

\begin{tabular}{cccc}
\hline \hline & $I$ & $N N$ & $N \Delta$ \\
\hline$T=0$ & 1 & ${ }^{3} S_{1}-{ }^{3} D_{1}$ \\
& & ${ }^{1} P_{1}$ & \\
$T=1$ & 2 & ${ }^{3} D_{2}$ & \\
& 0 & ${ }^{1} S_{0}$ & ${ }^{5} D_{0}$ \\
& 1 & ${ }^{3} P_{0}$ & ${ }^{3} P_{0}$ \\
& 2 & ${ }^{3} P_{1}$ & ${ }^{3} P_{1}-{ }^{5} P_{1}-\left({ }^{5} F_{1}\right)$ \\
& ${ }^{3} P_{2}-{ }^{3} F_{2}$ & ${ }^{3} P_{2}-{ }^{5} P_{2}-\left({ }^{3} F_{2}\right)-\left({ }^{5} F_{2}\right)$ \\
& ${ }^{1} D_{2}$ & ${ }^{5} S_{2}-{ }^{3} D_{2}-{ }^{5} D_{2}-\left({ }^{5} G_{2}\right)$ \\
\hline
\end{tabular}

large cutoff; however, the 5-channel results are physically not really significant, since they are far from convergence when a three-nucleon force is added.

The unifying calculations, the main object of this paper, take single $\Delta$-isobar excitation into account. The employed Hamiltonian is diagrammatically defined in Fig. 2. The processes (a)-(d) of Fig. 2 are parametrized by the force model A2 considered in Ref. [5] as characteristic for $\Delta$-isobar effects on trinucleon properties. That force model is an extension, an almost phaseequivalent extension of the Paris potential modified by explicit single $\Delta$-isobar excitations. The nucleon- $\Delta$ potentials of the processes (c) and (d) in Fig. 2 are put to zero. Their effect is investigated in Ref. [18] and found to be small. The Tucson-Melbourne three-nucleon force without a $\Delta$-pole contribution is used as process (e) of Fig. 2. The irreducible three-baryon forces with $\Delta$-isobar excitation in external legs as in the processes $(f)-(j)$ of Fig. 2 are neglected. The two-baryon partial waves considered in the solution of Eq. (4) are those of Ref. [5]; they are listed in Table IV. The full calculations are based on 6, 32, and 33 three-particle channels: (i) In 6channel calculations, the two-baryon interaction is allowed to act in all positive parity two-baryon partial waves with total pair momentum $I \leq 1$. Then ${ }^{1} S_{0}(N N)-{ }^{5} D_{0}(N \Delta)$ provides the only coupling of twonucleon to nucleon- $\Delta$ states and only one three-particle channel with a $\Delta$ isobar is to be added to the five purely nucleonic ones. (ii) In 32-channel calculations, the twobaryon interaction is taken to act in all two-baryon partial waves of Table IV. However, the $\Delta$ isobar is not allowed to be a spectator when two nucleons interact. Thus 14 three-particle channels with a $\Delta$ isobar are to be added to the 18 purely nucleonic ones. (iii) In 33-channel calculations, the two-baryon interaction is taken to act in all two-baryon partial waves of Table IV. The $\Delta$ isobar is allowed to be a spectator, however, only when two nucleons interact in a ${ }^{1} S_{0}$ state. Fifteen three-particle channels with a $\Delta$ isobar are to be added to the 18 purely nucleonic ones. We only consider the calculations based on 33 three-particle channels well converged with respect to channel truncation. A very careful investigation on the convergence issue and the confirmation of that fact are given in Ref. [19].

The results of that unified calculations are listed in Tables V and VI and displayed in Figs. 4 and 5. They confirm the reference calculations that the TucsonMelbourne three-nucleon force without its $\Delta$-pole part still makes a non-negligible contribution to the triton binding energy. That contribution is, however, much smaller compared with the effect of the full TucsonMelbourne three-nucleon force with a $\Delta$-pole part. Furthermore, the dependence of the binding-energy results on the cutoff parameter $\Lambda$ for the $\pi N N$ vertex in the Tucson-Melbourne force is considerably reduced. Table $\mathrm{V}$ shows that the results based on the two different sets of strength parameters for the Tucson-Melbourne force without a $\Delta$-pole part are qualitatively quite similar. In fact, at the recommended cutoff value $\Lambda=5.8 \mu$, the theoretical predictions of the triton binding energy, based on $\Delta$-isobar dynamics and the additional three-nucleon force, become close to the experimental value of -8.48 $\mathrm{MeV}$. Tables III and $\mathrm{V}$ together indicate that the inclusion of explicit $\Delta$ components in the trinucleon wave function leads to a moderate increase for the triton binding energy of the same size as observed in calculations without an irreducible three-nucleon force; that particular result is almost independent of the value for the cutoff

TABLE V. Triton binding energy in MeV for the force model of Fig. 2 with explicit single $\Delta$-isobar excitation and an irreducible three-nucleon interaction. When no irreducible three-nucleon force is added, the force model of Fig. 2 becomes-in the employed parametrization-identical with the coupled-channel force model A2 of Ref. [5]. In the table the results for the force model A2 are indicated by Paris $+\Delta$ (A2), since A2 is an extension of the Paris potential with single $\Delta$-isobar excitation. The added irreducible three-nucleon forces are based on the Tucson-Melbourne force, from which the $\Delta$ pole contribution is subtracted according to the two different sets of reduced strength parameters in Table I. The calculations refer to 6, 32, and 33 three-particle channels and to various cutoff parameters $\Lambda$ in the $\pi N N$ vertex of Eq. (3) in the employed irreducible three-nucleon forces.

\begin{tabular}{|c|c|c|c|c|c|c|}
\hline & & No TM- $\Delta$ & $\begin{array}{c}\Lambda=4.1 \mu \\
\mathrm{TM}-\Delta 1\end{array}$ & $\begin{array}{c}\Lambda=5.8 \mu \\
\mathrm{TM}-\Delta 1\end{array}$ & $\begin{array}{c}\Lambda=7.1 \mu \\
\text { TM- } \Delta 1\end{array}$ & $\begin{array}{c}\Lambda=5.8 \mu \\
\text { TM- } \Delta 2\end{array}$ \\
\hline Paris $+\Delta$ (A2) & 6 & -6.83 & -6.62 & -7.02 & -10.49 & -7.09 \\
\hline Paris $+\Delta$ (A2) & 32 & -7.70 & -7.60 & -8.17 & -9.48 & -8.38 \\
\hline Paris $+\Delta$ (A2) & 33 & -7.72 & -7.63 & -8.20 & -9.49 & -8.41 \\
\hline
\end{tabular}


TABLE VI. Triton properties derived from various force models based on the Paris potential. The binding energy $E$ and probabilities (in \%) for the wave function components with total orbital angular momentum 0 , i.e., $P(S)$ of totally symmetric orbital symmetry and $P\left(S^{\prime}\right)$ of mixed symmetry, with total orbital angular momenta 1 and 2, i.e., $P(P)$ and $P(D)$, and with $\Delta$-isobar content, i.e., $P(\Delta)$, are listed. The calculations with purely nucleonic wave function components refer to 18 three-particle channels and the calculations with additional single $\Delta$-isobar components to 33 three-particle channels. The cutoff parameter $\Lambda$ in the $\pi N N$ vertex of the Tucson-Melbourne three-nucleon force and in its modifications is $5.8 \mu$.

\begin{tabular}{lcccccc}
\hline \hline & $E(\mathrm{MeV})$ & $P(S)$ & $P\left(S^{\prime}\right)$ & $P(P)$ & $P(D)$ & $P(\Delta)$ \\
\hline Paris & -7.38 & 90.07 & 1.43 & 0.07 & 8.43 & 0.00 \\
Paris $+\Delta$ (A2) & -7.72 & 87.53 & 1.49 & 0.09 & 8.47 & 2.43 \\
Paris + $(\mathrm{A} 2)+(\mathrm{TM}-\Delta 1)$ & -8.20 & 87.52 & 1.35 & 0.11 & 8.56 & 2.47 \\
Paris + $\Delta$ (A2)+(TM- $\Delta 2)$ & -8.41 & 87.40 & 1.29 & 0.12 & 8.68 & 2.51 \\
Paris + TM & -9.08 & 89.85 & 1.00 & 0.18 & 8.97 & 0.00 \\
\hline \hline
\end{tabular}

parameter $\Lambda$ in the $\pi N N$ vertex of the Tucson-Melbourne force. Table VI lists wave function properties arising from different calculations.

Our objective was to unify the existing triton calculations of Refs. [1-3] with an irreducible three-nucleon force and those of Refs. [4,5] based on $\Delta$-isobar dynamics. On purpose, we therefore adopted the underlying hadronic concepts of Refs. [1-3], on the one hand, and of Refs. $[4,5]$, on the other hand, without essential changes. That idea had to result in inconsistencies for the parametrization of the employed force model of Fig. 2. Examples for those inconsistencies are the following: (i) The Tucson-Melbourne three-nucleon force used in Refs. $[1-3]$ is based on pion exchange, whereas Refs. [4,5] base the transition potentials to nucleon- $\Delta$ states on pion and rho exchange. (ii) The employed form of the TucsonMelbourne three-nucleon force in Eq. (2) uses a dipole form factor for each pion exchange, whereas Refs. [4,5] use a monopole form factor instead. (iii) The actual values for the cutoff mass $\Lambda$ in the form factors for the Tucson-Melbourne three-nucleon force and for the transition potential are physically inequivalent, even when allowance is made for the different multipole character of the form factors according to (ii). References [4,5] use a larger cutoff mass for the transition potential which would correspond to $\Lambda=\sqrt{2} 5.8 \mu=8.2 \mu$ in a dipole form factor. However, Ref. [12] demonstrates that the $\Delta$ isobar-induced effects on the triton binding are small in that parameter region in which the Tucson-Melbourne three-nucleon force shows rather singular behavior with dramatically cutoff dependent binding-energy results. (iv) In the Tucson-Melbourne force of Eq. (2), the pion exchange is of ordinary pion range. This is the reason why the force model A1 whose pion exchange is also of ordinary range is used in the scheme (1) of Sec. II for subtracting the $\Delta$-pole part from the Tucson-Melbourne force. In contrast, the force model A2, considered as especially characteristic for $\Delta$-induced effects and taken for the present unifying calculations, employs a transition potential with partially modified pion range, since in a time-ordered picture the simultaneous propagation of a pion and a $\Delta$ isobar corresponds to a mass effectively larger than that of the pion.

We think that those inconsistencies, tolerated for the present limited purpose of unification, do not cloud our conclusions. In fact, inconsistencies of that sort have occurred generally in all previous calculations, when a three-nucleon force is added to a Hamiltonian with twonucleon potentials.

We conclude that $\Delta$ excitation seems to be indeed the most important process contained in the TucsonMelbourne two-pion exchange three-nucleon force. Thus the explicit treatment of the $\Delta$ isobar as an additional nuclear constituent is advisable. The comparison with calculations based on the full Tucson-Melbourne threenucleon force confirm the observation of Ref. [12] that static approximations on the propagation of the $\Delta$ isobar can lead to a severe overestimate of $\Delta$-induced effects in nuclear properties.

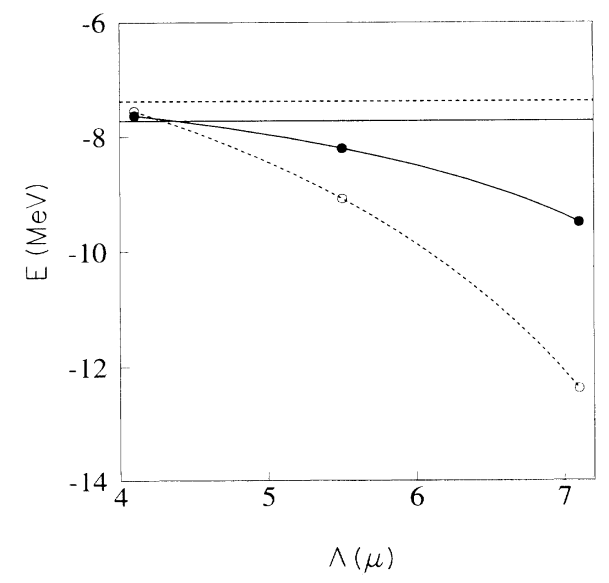

FIG. 4. Triton binding energy in $\mathrm{MeV}$ vs the cutoff parameter $\Lambda$ in the $\pi N N$ form factor of Eq. (3) for the TucsonMelbourne three-nucleon force in units of the charged pion mass $\mu$. Solid circles represent the results of the full unifying calculation with explicit $\Delta$ isobars taken into account in 33 channels and with the modified Tucson-Melbourne force TM$\Delta 1$ of Table I added. Open circles represent the binding-energy results for purely nucleonic 18-channel calculations with the Paris potential and the full Tucson-Melbourne three-nucleon force without subtraction of $\Delta$ contributions. Curves through the symbols are meant to guide the eye. The horizontal solid line indicates the binding energy obtained with $\Delta$-isobar excitation, but without irreducible three-nucleon force, and the dashed line the one obtained from the Paris potential without three-nucleon force. 


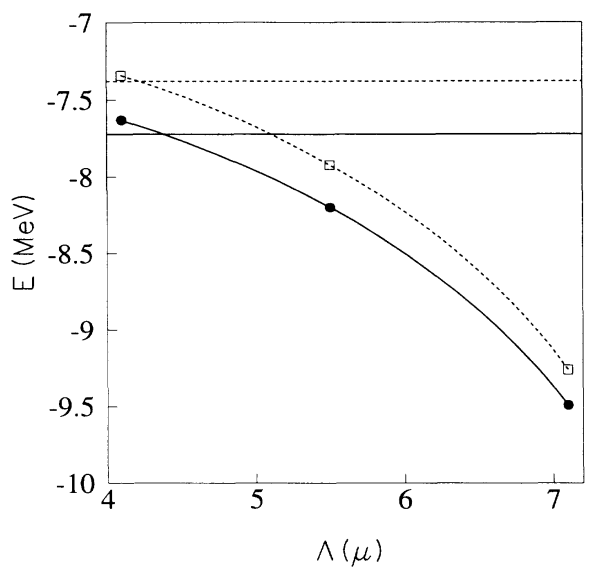

FIG. 5. Triton binding energy in $\mathrm{MeV}$ vs the cutoff parameter $\Lambda$ in the $\pi N N$ form factor of Eq. (3) for the modified Tucson-Melbourne three-nucleon force TM- $\Delta 1$ of Table $I$ in units of the charged pion mass $\mu$. Solid circles represent the results of the full unifying calculation with explicit $\Delta$ isobars taken into account in 33 channels. Open boxes represent the binding-energy results for purely nucleonic 18-channel calculations with the Paris potential. In both cases the modified Tucson-Melbourne three-nucleon force $T M-\Delta 1$ is added. Curves through the symbols are meant to guide the eye. The horizontal solid line indicates the binding energy obtained with $\Delta$-isobar excitation, but without irreducible three-nucleon force, and the dashed line the one obtained from the Paris potential without three-nucleon force. Compared with Fig. 4, the difference in scale should be noted; the two solid curves are the same as in Fig. 4.

\section{ACKNOWLEDGMENTS}

The authors thank S. A. Coon, B. H. J. McKellar, and M. T. Peña for interesting discussions on the TucsonMelbourne force in general and S. A. Coon and M. T. Peña in particular for their help with respect to the subtraction schemes of the $\Delta$ contributions. A. S. thanks C. Chandler and B. F. Gibson for their kind hospitality during a stay at the University of New Mexico, Albuquerque, and at the Los Alamos National Laboratory. P.U.S. thanks the Nuclear Theory Group of the University of Washington for the hospitality extended to him during a sabbatical stay. This work is funded by the Deutsche Forschungsgemeinschaft (DFG) under Contract No. Sa 247/7-1 and by the U.S. Department of Energy under Contract No. DE-FG06-88ER40427. The culations were performed at "Regionales Rechenzentrum für Niedersachsen."

\section{APPENDIX}

The full expression for the matrix elements of the permutation operator $P$ is given in the basis of the threeparticle states $|p q v\rangle$. In these states the (23) pair is antisymmetrized, though the index 1 on the ket identifying the antisymmetrized pair will be suppressed. However, the suppressed index reappears in the matrix elements for clarifying the meaning of some quantum numbers.

The matrix elements can be decomposed in the form

$$
\begin{aligned}
& \left\langle p q v|P| p^{\prime} q^{\prime} v^{\prime}\right\rangle \\
& =\int_{-1}^{+1} d x \frac{\delta\left(p-p_{1}^{v v^{\prime}}\left(q, q^{\prime}, x\right)\right)}{p^{L+2}} \\
& \quad \times \frac{\delta\left(p^{\prime}-p_{2}^{v v^{\prime}}\left(q, q^{\prime}, x\right)\right)}{p^{L^{\prime}+2}} G_{v v^{\prime}}\left(q, q^{\prime}, x\right),
\end{aligned}
$$

with

$$
\begin{aligned}
& p_{1}^{v v^{\prime}}\left(q, q^{\prime}, x\right)=\left[(\gamma q)^{2}+q^{\prime 2}+2 \gamma q q^{\prime} x\right]^{1 / 2}, \\
& p_{2}^{v v^{\prime}}\left(q, q^{\prime}, x\right)=\left[q^{2}+\left(\alpha q^{\prime}\right)^{2}+2 \alpha q q^{\prime} x\right]^{1 / 2}, \\
& x=\hat{\mathbf{q}} \cdot \hat{\mathbf{q}}^{\prime} .
\end{aligned}
$$

The constants $\alpha$ and $\gamma$ depend through the discrete quantum numbers $B, b, B^{\prime}$, and $b^{\prime}$ on the channels $v$ and $v^{\prime}$ according to

$$
\begin{gathered}
\alpha=\frac{\frac{1}{2}-r_{m} b}{1-r_{m} B^{\prime}}, \\
\gamma=\frac{\frac{1}{2}-r_{m} b^{\prime}}{1-r_{m} B},
\end{gathered}
$$

with the mass ratio

$$
r_{m}=\frac{m_{\Delta}-m_{N}}{m_{\Delta}+m_{N}} .
$$

This is the reason why the momenta $p_{1}^{\nu v^{\prime}}$ and $p_{2}^{\nu v^{\prime}}$ carry both channel labels.

The reduced matrix elements $G_{v v^{\prime}}\left(q, q^{\prime}, x\right)$ are given by

$$
\begin{aligned}
& G_{v v^{\prime}}\left(q, q^{\prime}, x\right)=\frac{1}{\left[\left(1+B^{2}\right)\left(1+B^{\prime 2}\right)\right]^{1 / 2}} \sum_{k} P_{k}(x) \sum_{L_{1}+L_{2}=L} \sum_{L_{1}^{\prime}+L_{2}^{\prime}=L^{\prime}} q^{L_{1}+L_{1}^{\prime} q^{\prime L_{2}+L_{2}^{\prime}}} \\
& \times\left\{g_{v(2,3) v^{\prime}\left(2^{\prime}, 3^{\prime}\right)}^{k L_{1} L_{1}^{\prime} L_{2} L_{2}^{\prime}} \delta_{b_{1} b_{3}^{\prime}} \delta_{b_{2} b_{1}^{\prime}} \delta_{b_{3} b_{2}^{\prime}}-(-)^{L-S-T+s_{2}+s_{3}+t_{2}+t_{3}} g_{v(3,2) v^{\prime}\left(2^{\prime}, 3^{\prime}\right)}^{k L_{1} L_{b_{1}^{\prime} L^{\prime} L_{2} L_{3}^{\prime}}^{\prime}} \delta_{b_{2} b_{2}^{\prime}} \delta_{b_{3} b_{1}^{\prime}}\right. \\
& -(-)^{L^{\prime}-S^{\prime}-T^{\prime}+s_{2}^{\prime}+s_{3}^{\prime}+t_{2}^{\prime}+t_{3}^{\prime}} g_{v(2,3) v^{\prime}\left(3^{\prime}, 2^{\prime}\right)}^{k L_{1} L^{\prime} L_{2} L_{1}^{\prime}} \delta_{b_{2}^{\prime} b_{2}^{\prime}} \delta_{b_{2} b_{1}^{\prime}} \delta_{b_{3} b_{3}^{\prime}}
\end{aligned}
$$

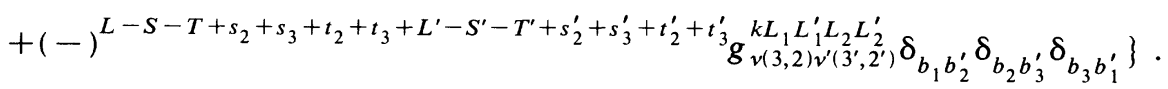


In Eq. (A8), $P_{k}(x)$ are the ordinary Legendre polynomials. The factors $g_{v(2,3) v^{\prime}\left(2^{\prime}, 3^{\prime}\right)}^{k L_{1} L^{\prime} L_{2} L^{\prime}}$ are of purely geometrical nature, i.e.,

$$
\begin{aligned}
& g_{v(2,3) v^{\prime}\left(2^{\prime}, 3^{\prime}\right)}^{k L_{1} L_{1}^{\prime} L_{2} L_{2}^{\prime}}=(-)^{L+S^{\prime}+T^{\prime}+s_{1}+s_{3}+2 s_{2}+t_{1}+t_{3}+2 t_{2}} \delta_{\not \gamma^{\prime}} \delta_{\partial_{z} g_{z}^{\prime}} \delta_{\mathcal{T} \mathcal{T}^{\prime}} \delta_{\mathcal{T}_{z} \tau_{z}^{\prime}} \\
& \times \hat{I} \hat{I}^{\prime} \hat{j} \hat{j}^{\prime} \hat{L} \hat{L}^{\prime} \hat{S} \hat{S}^{\prime} \hat{l} \hat{l}^{\prime} \hat{T} \hat{T}^{\prime} \frac{1}{2} \hat{k}^{2} \gamma^{L_{1}} \alpha^{L_{2}^{\prime}}\left(\frac{(2 L+1) !\left(2 L^{\prime}+1\right) !}{\left(2 L_{1}\right) !\left(2 L_{2}\right) !\left(2 L_{1}^{\prime}\right) !\left(2 L_{2}^{\prime}\right) !}\right)^{1 / 2}\left\{\begin{array}{ccc}
t_{2} & t_{3} & T \\
t_{1} & T & T^{\prime}
\end{array}\right\}
\end{aligned}
$$

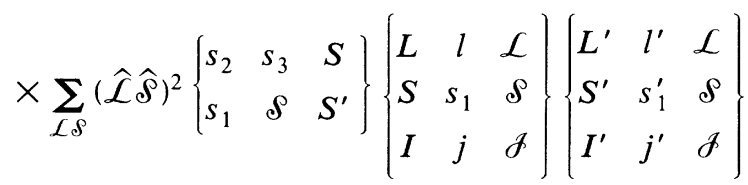

$$
\begin{aligned}
& \times \sum_{f f^{\prime}}\left\langle L_{1} 0 l 0 \mid f 0\right\rangle\left\langle L_{2}^{\prime} 0 l^{\prime} 0 \mid f^{\prime} 0\right\rangle\left\langle k 0 L_{2} 0 \mid f^{\prime} 0\right\rangle\left\langle k 0 L_{1}^{\prime} 0 \mid f 0\right\rangle \\
& \times\left\{\begin{array}{ccc}
L_{1} & L_{2} & L \\
\mathcal{L} & l & f
\end{array}\right\}\left\{\begin{array}{ccc}
L_{2}^{\prime} & L_{1}^{\prime} & L^{\prime} \\
\mathcal{L} & l^{\prime} & f^{\prime}
\end{array}\right\}\left\{\begin{array}{ccc}
f & L_{2} & \mathcal{L} \\
f^{\prime} & L_{1}^{\prime} & k
\end{array}\right\},
\end{aligned}
$$

where $\hat{x} \equiv \sqrt{2 x+1}$. The more explicit notation $v(j, k)$ for the channel label $v$ in the geometric factors of Eq. (A9) should be a reminder that the particle labels in the pair are $j$ and $k$ and they should indicate the order in which the spins and isospins of the pair particles $j$ and $k$ are coupled.

[1] C. R. Chen, G. L. Payne, J. L. Friar, and B. F. Gibson, Phys. Rev. C 33, 1740 (1986).

[2] T. Sasakawa and S. Ishikawa, Few-Body Syst. 1, 3 (1986).

[3] A. Bömelburg, Phys. Rev. C 34, 14 (1986).

[4] Ch. Hajduk and P. U. Sauer, Nucl. Phys. A322, 329 (1979).

[5] Ch. Hajduk, P. U. Sauer, and W. Strueve, Nucl. Phys. A405, 581 (1983).

[6] H. Pöpping, P. U. Sauer, and Zhang Xi-Zhen, Nucl. Phys. A474, 557 (1987).

[7] S. A. Coon, M. D. Scadron, P. C. McNamee, B. R. Barrett, D. W. E. Blatt, and B. H. J. McKellar, Nucl. Phys. A317, 242 (1979).

[8] S. A. Coon and M. T. Peña, Few-Body Syst, Suppl. 6, 242 (1992).

[9] S. A. Coon and W. Glöckle, Phys. Rev. C 23, 1790 (1981).

[10] A. Bömelburg, Ph.D. thesis, Universität Bochum, 1986 (unpublished).
[11] H. T. Coelho, T. K. Das, and M. R. Robilotta, Phys. Rev. C 28, 1812 (1983).

[12] Ch. Hajduk, P. U. Sauer, and Shin Nan Yang, Nucl. Phys. A405, 605 (1983).

[13] S. A. Coon (private communication).

[14] A. Stadler, W. Glöckle, and P. U. Sauer, Phys. Rev. C 44, 2319 (1991).

[15] R. V. Reid, Ann. Phys. (N.Y.) 50, 411 (1968).

[16] M. Lacombe, B. Loiseau, J. M. Richard, R. Vinh Mau, J. Côté, P. Pirès, and R. de Tourreil, Phys. Rev. C 21, 861 (1980).

[17] J. L. Friar, B. F. Gibson, G. L. Payne, and S. A. Coon, Few-Body Syst. 5, 13 (1988).

[18] M. T. Peña, H. Henning, and P. U. Sauer, Phys. Rev. C 42, 855 (1990).

[19] A. Pickelsimer, R. A. Rice, and R. Brandenburg, Phys. Rev. C 44, 1354 (1991). 